\title{
A new urticarial rash, diarrhoea and refractory hypotension in a man with a history of migraines
}

\author{
Authors: Lucy Lamb ${ }^{A}$ and David Lowe ${ }^{B}$
}

We present the case of a man with a history of migraines treated with propanolol, referred with a rash, diarrhoea, vomiting and hypotension. Our case highlights how prior betablocker use may prolong anaphylaxis and cause refractory hypotension.

KEYWORDS: Beta-blockers, prolonged anaphylaxis, tryptase, refractory hypotension

\section{Case presentation}

A 43-year-old male IT consultant presented to the accident and emergency department of the Royal Free Hospital with a new rash, vomiting, diarrhoea and profound postural hypotension unresponsive to fluids. He had a long history of migraines for which he was taking prophylactic propranolol; on the day prior to admission, he had received a repeat prescription of propranolol from a new manufacturer, which contained cellulose, and eaten a meal containing cooked salmon.

On review by the medical team, he reported some facial and finger swelling and dizziness on sitting. Examination revealed lip and finger swelling and a widespread urticarial rash over the trunk and limbs (Fig 1). Measurement of lying and sitting blood pressures revealed a postural drop in systolic blood pressure of greater than $50 \mathrm{mmHg}$. The remainder of the examination was unremarkable. His blood tests were normal apart from a neutrophilia (white blood cell count $12.69 \times 10^{9} / \mathrm{L}$, neutrophils $10.89 \times 109 / \mathrm{L})$ and elevated C-reactive protein $(19 \mathrm{mg} / \mathrm{L}$ rising to $81 \mathrm{mg} / \mathrm{L}$ within 8 hours).

\section{Differential diagnosis}

This patient had been referred with refractory hypotension, diarrhoea and vomiting and a new rash, thought to be associated with a recent change in his beta-blocker manufacturer. His symptoms initially were thought to be due to viral gastroenteritis but this did not explain the new urticarial

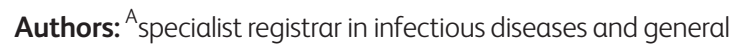
(internal) medicine, Royal Free London NHS Foundation Trust, London, UK; ${ }^{B}$ consultant in immunology and acute medicine, Institute of Immunity and Transplantation, University College London, London, UK

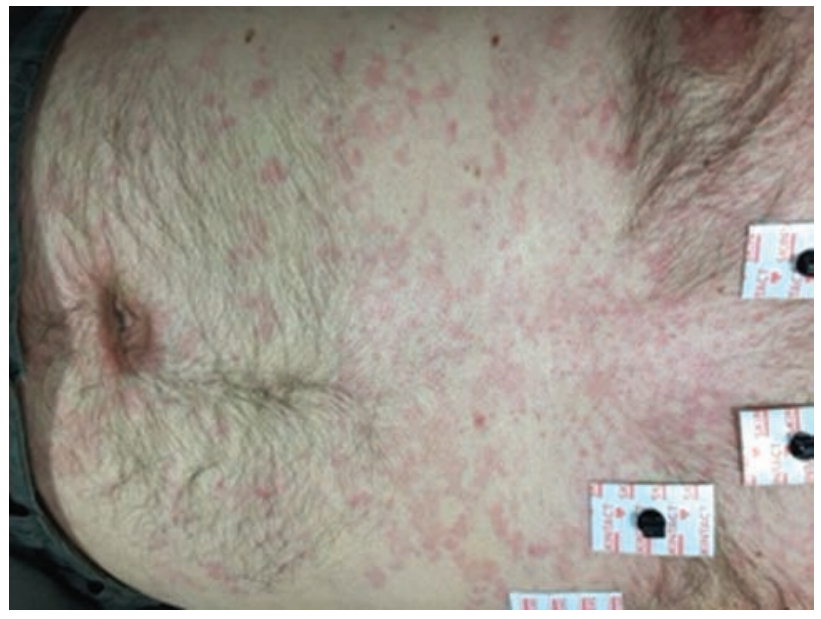

Fig 1. Urticarial rash visible on the trunk.

rash. With this in mind, it was thought that he showed a delayed anaphylactic response to cellulose or scombroid poisoning from salmon ingestion the day before. During the course of his illness, he developed a fever and bacterial sepsis was considered as another differential diagnosis for his refractory hypotension. To confirm the diagnosis, we proceeded to measure the mast cell tryptase response and sent blood and stool for culture and viral polymerase chain reaction (PCR).

\section{Case progression}

Initially, the patient was treated with hydrocortisone, chlorpheniramine and intramuscular adrenaline $(500 \mathrm{mcg}$ of 1:1000 concentration). At first, he showed a good response with a reduction in facial swelling, fading of the rash and improvement in blood pressure. However, the response was limited and he subsequently experienced two further episodes of facial swelling and hypotension both requiring further doses of intramuscular adrenaline.

During the admission he developed a fever and intravenous ceftriaxone was commenced to treat potential bacterial sepsis, although subsequent blood cultures were negative. Further blood tests showed an initial rise in mast cell trypase (Fig 2) supporting a diagnosis of anaphylaxis rather than scombroid poisoning. Additionally, PCR of stool was positive for norovirus. 


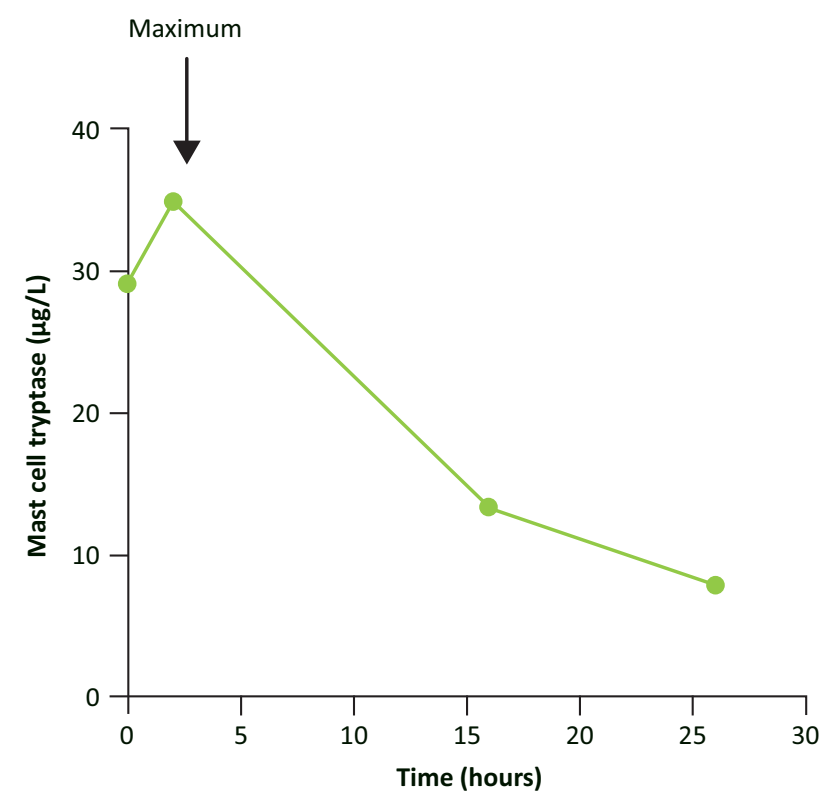

Fig 2. Response seen by the mast cell tryptase during the course of the illness.

\section{Discussion}

In this case report, we describe the delayed presentation and protracted reversal of an anaphylactic reaction in a patient treated with beta-blockers. We show how mast cell tryptase measurement can be useful in the follow-up of suspected anaphylactic reactions, although clinicians must be mindful that collection should not delay resuscitation. ${ }^{1}$ It is likely in this case that the symptoms were exacerbated both by prior use of beta-blockers and a concomitant norovirus infection. Scombroid poisoning was considered as a differential diagnosis as it can lead to 'allergic-like' symptoms, including an urticarial rash, vomiting, diarrhoea and hypotension. This is a complex of symptoms caused by biogenic amines - mainly histamine contained in seafood. However, it was felt that this was not the cause because it typically results in no significant rise in mast cell tryptase $e^{2}$ and would usually affect all of the people who ingested the fish. We also considered whether this was a biphasic anaphylactic reaction but this was thought unlikely because of the failure to fully respond before requiring more treatment, the absence of a persistent or second rise in mast cell tryptase and complete resolution of symptoms within 36 hours.

Beta-blockers inhibit both the endogenous and exogenous administration of epinephrine leading to a more severe, protracted and difficult to treat anaphylaxis. ${ }^{3}$ In our case, it appears that the patient experienced an allergic reaction to a new cellulose-containing beta-blocker preparation. He was slow to treat and resuscitate, plausibly because of a longstanding history of beta-blocker use and gastroenteritis caused by norovirus infection. His management consisted of the administration of adrenaline and initiation of aggressive fluid resuscitation, ${ }^{1}$ which in this case was successful. If adrenaline and fluids fail to restore the blood pressure, other agents like glucagon could be considered. ${ }^{4-6}$ Patients who have suffered a suspected anaphylaxis should be observed closely and referred to an appropriate allergy service for follow-up. Adverse drug reactions should be reported to the Medicines and Healthcare products Regulatory Agency (www.mhra.gov.uk). Clinicians should also be aware of biphasic reactions (incidence ranges from 1-20\%), a reoccurrence of symptoms within 72 hours following apparent complete recovery of anaphylaxis: management is the same as for the first episode. It has been suggested that patients should be observed for 24 hours after recovery in view of this possibility. ${ }^{7-9}$

\section{Key learning points}

$>$ Consider a delay in presentation of anaphylaxis and response to intramuscular adrenaline in patients who have been taking beta-blockers.

> Allergies to medicines may occur simply because of a change in manufacturer.

> Mast cell tryptase levels may be used to diagnose acute anaphylaxis and exclude other differentials, like scombroid poisoning, but measurement should not delay treatment.

$>$ Consider other causes of refractory hypotension, including sepsis.

> Patients may require observation for up to 24 hours to exclude a biphasic reaction.

\section{Conflicts of interest}

The authors declare no conflicts of interest.

\section{Acknowledgements}

Written informed consent was obtained from the patient to publish the clinical details and images in this article.

\section{References}

1 Advanced Life Support, 7th edn. London: Resuscitation Council UK, 2016.

2 Ricci G, Zannoni M, Cigolini D et al. Tryptase serum level as a possible indicator of scombroid syndrome. Clin Toxicol 2010;48:203-6.

3 Muller UR, Haeberli G. Use of beta-blockers during immunotherapy for Hymenoptera venom allergy. J Allergy Clin Immunol 2005;115:606-10.

4 Campbell RL, Li JT, Nicklas RA et al. Emergency department diagnosis and treatment of anaphylaxis: a practice parameter. Ann Allergy Asthma Immunol 2014;113:599-608.

5 Lang DM, Alpern MB, Visintainer PF et al. Elevated risk of anaphylactoid reaction from radiographic contrast media is associated with both beta-blocker exposure and cardiovascular disorders. Arch Intern Med 1993;153:2033-40.

6 Toogood JH. Beta-blocker therapy and the risk of anaphylaxis. CMAJ 1987;136:929-933.

7 National Institute for Health and Care Excellence. Anaphylaxis: assessment and referral after emergency treatment. NICE clinical guideline 134. Manchester: NICE, 2011.

8 Lieberman P. Biphasic anaphylactic reactions. Ann Allergy Asthma Immunol 2005;95:217-26.

9 Limb SL, Starke PR, Lee CE et al. Delayed onset and protracted progression of anaphylaxis after omzlizumab administration in patients with asthma. J Allergy Clin Immunol 2007;120:1373-7. 